\title{
Gene Action and Combining Ability for Yield and Quality Traits in Rice (Oryza sativa L.) using Diallel Analysis
}

\author{
K. Rukmini Devi*, V. Venkanna, B. Satish Chandra and Y. Hari \\ Regional Agricultural Research Station, Mulug road 506007, Warangal, Professor \\ Jayashankar Telangana State Agricultural University, Telangana, India \\ *Corresponding author
}

\section{A B S T R A C T}

\section{Keywords}

Diallel analysis,

Combining ability,

Rice, Quality,

Additive and non-

additive gene

effects

\section{Article Info}

Accepted:

20 December 2017

Available Online:

10 January 2018
Eight high yielding rice genotypes along with their twenty eight crosses which effected in a diallel fashion excluding reciprocals were subjected to combining ability which aimed at elucidating the genetic system underlying the inheritance of yield and quality traits. The study revealed predominance of non-additive gene action for most of the yield components and quality traits except straw yield/plant, which was under the control of additive gene action. NLR 40024, MTU 1075 and HKR08-62 were identified as good general combiners for grain yield/plant, panicle length, panicle weight, straw yield/plant, harvest index, head rice recovery, kernel length after cooking, water uptake and alkali spreading value. The crosses Kavya $\times$ HKR08-62, Gontrabidhan $\times$ HKR08-62, Kavya $\times$ MTU 1075 have shown significantly favorable SCA effects for grain yield, yield components and quality traits. Majority of the crosses with high SCA effects involved with high $\times$ low combinations indicating additive $\times$ dominance, dominance $\times$ dominance type of gene interactions for expression of traits. To explore both additive and non-additive type of gene action, biparental mating among selected crosses or use of selection procedure such as diallel selective mating may be followed.

\section{Introduction}

Rice is the world's second most important cereal crop and staple food for more than 60 $\%$ of the global population. To meet the food demand of the growing population and to achieve food security in the country, the production levels need to be increased by 2 million tons every year. As there is no scope to increase the cultivable area the only alternative is to improve the genetic potential. Plant breeders continuously refine and improve genetic traits of new varieties required. One of the main problems for improving high yielding varieties is to select suitable parents and hybrids derived from the crosses between them. In order to formulate efficient breeding programmes, for improvement of yield and quality traits it is essential to characterize the nature and mode of gene action. A sound breeding methodology rests on a correct understanding of the gene effects involved. The combining ability analysis provides the information about the parents for hybridization. Efficient identification of superior cross combinations 
is another issue in hybrid breeding. Phenotypic selection of promising parental lines can be performed either based on GCA and SCA is most important for predicting hybrid performance based on GCA effects (Fischer et al., 2008).

Diallel analysis is one of the most powerful tools for estimating the general combining ability (GCA) of parents and selection of desirable parents and crosses with high specific combining ability (SCA) for exploitation of heterosis and to get the knowledge of gene effects and variance components. Hence the present study was undertaken to assess the combining ability of promising eight rice genotypes using half diallel analysis.

\section{Materials and Methods}

The material comprised of eight high yielding, photoinsensitive and diverse varieties viz., Kavya, Gontrabidhan, BPT 5204, R15562577, MTU 1075, WGL 915, NLR 40024 and HKR08-62 were crossed in diallel fashion without reciprocals. The 36 entries i.e. 28 crosses and 8 parents were grown in a randomized block design with two replications at Regional Agricultural Research Station, Warangal during Kharif 2016. Single seedling per hill was transplanted at a spacing of 20 $\mathrm{x} 15 \mathrm{~cm}$ between the rows and plants. Each entry consists of two rows of $3 \mathrm{~m}$ length. All the recommended cultural practices were followed to obtain healthy crop.

Observations were recorded on 10 randomly selected plants without border effect for twenty five yield and quality traits except days to $50 \%$ flowering which was taken on plot basis, plant height $(\mathrm{cm})$, panicle length $(\mathrm{cm})$, effective tillers/plant, flag leaf length $(\mathrm{cm})$, flag leaf width $(\mathrm{cm})$, panicle weight $(\mathrm{g})$, panicle density, filled grains/panicle, test weight (g), grain yield/plant (g), straw yield/plant (g), harvest index, hulling percent, milling percent, head rice recovery (\%), kernel length $(\mathrm{mm})$, kernel width $(\mathrm{mm})$, length/breadth ratio, kernel length after cooking $(\mathrm{mm})$, kernel width after cooking $(\mathrm{mm})$, kernel elongation ratio, volume expansion ratio, water uptake $(\mathrm{ml})$ and alkali spreading value. Data on hulling and milling was taken with the help of SATAKE company make laboratory huller and polisher. Data on head rice recovery was recorded. Kernel length and kernel width of 20whole milled rice were measured by means of dial caliper and length/breadth ratio was computed as per Murthy and Govida Swamy (1967).

Kernel elongation ratio was determined by soaking $5 \mathrm{~g}$ of whole milled rice in $12 \mathrm{ml}$ distilled water for 10 minutes and later cooked for 15 minutes in water bath. Observations on length and breadth of cooked kernel and kernel elongation ratio were recorded with the help of graph sheet to quantify cooking traits. Volume expansion ratio, water uptake and alkali spreading value were estimated by following standard procedures. The combining ability analysis was carried out as per Griffing (1956), method-2 (Model-I).

\section{Results and Discussion}

Analysis of variance for combining ability (Table 1) showed significant differences among 36 genotypes for all the characters studied. Mean squares due to general combining ability (GCA) were significant for all the character except kernel elongation ratio, whereas specific combining ability (SCA) were significant for all the yield and quality traits studied. This suggests importance of both additive and non-additive gene effects in the material under study. Hence any approach that facilitates simultaneous exploitation of both additive and non-additive gene effects would be more desirable for the improvement of the traits. 
The estimates of the components due to GCA and SCA effects and their ratio showed that non additive gene effect was higher than additive effect for all the yield and quality characters except straw yield/plant for which additive component may be predominant. Occurrence of both additive and non-additive gene effects with predominance of nonadditive gene action for yield and quality traits in rice were reported by Rukmini Devi, et al., (2014 a), Verma and Srivastava (2004) and Vanaja, et al., (2003). also found that GCA and SCA effects were highly significant for days to $50 \%$ flowering, plant height, effective tillers, panicle length, filled grains per panicle and test weight, while Ram kishor et al., (2017) for plant height, effective tillers, flag leaf length, flag leaf width, panicle length, test weight and grain yield per plant indicating prevalence of additive and non-additive effects in the inheritance of traits, as well as greater importance of non-additive gene action.

The GCA effect which determines the average performance of a parent in crosses and is an estimate of its $F_{1}$ breeding value determined for each parent (Table 2). The selection of suitable parents for hybridization is one of the most important steps in a breeding programme to get best combination in $\mathrm{F}_{1}$ hybrids or segregating generation. The GCA is controlled by additive genes which is fixable. It provides information on the choice of parents in terms of expected performance of their progenies.

The genotype NLR 40024 was found to be good general combiner and possessed high GCA effects for important yield components like panicle length, panicle weight, panicle density, test weight, straw yield/plant, harvest index, head rice recovery, kernel length after cooking, water uptake and alkali spreading value. Another culture HKR08-62 was quite promising for effective tillers, flag leaf length (short flag leaf), panicle weight and kernel width after cooking (negative direction) and R1556-2577 for days to 50\% flowering (earliness), panicle length and kernel length, while MTU 1075 for grain yield/plant, filled grains per panicle, panicle length, kernel length, kernel width and kernel width after cooking in desired direction and length/breadth ratio. The genotype WGL 915 was good for effective tillers, straw yield per plant, harvest index and head rice recovery.

The negative estimates of GCA effect are desirable for earliness, plant height, flag leaf length and flag leaf width. Among the parents studied BPT 5204 proved to be good combiner for earliness, short stature, flag leaf length, flag leaf width and panicle density. For effective tillers HKR08-62 and WGL 915; for panicle length, panicle weight and panicle density R15556-2577, MTU 1075 and NLR 40024; for filled grains per panicle Kavya and MTU 1075; for test weight all the parents except BPT5204 exhibited significant GCA. For quality traits, WGL 915, Kavya and NLR 40024 for milling percent and NLR40024, WGL 915 and HKR08-62 recorded significant GCA values for head rice recovery. None of the parents were good general combiners for all the desirable traits studied.

Specific combining ability is the deviation from the performance predicted on the basis of general combining ability of the parents and it is due to non-additive gene effect. The usefulness of a particular cross in exploiting heterosis is judged by specific combining ability effects (Table 3). It is an important criterion for the evaluation of hybrids. Significant SCA effect for grain yield/plant were recorded by nine hybrids Kavya $\times$ HKR08-62, Gontrabidhan $\times$ HKR08-62, Kavya $\times$ MTU 1075, BPT $5204 \times$ R15562577, kavya $\times$ BPT 5204, Gontrabidhan $\times$ MTU 1075, BPT $5204 \times$ WGL 915, R1556$2577 \times$ HKR08-62 and WGL $915 \times$ HKR0862. 
Table.1 Analysis of variance for combining ability for yield, yield components and quality traits in $8 \times 8$ diallel analysis using Griffing's method-2

\begin{tabular}{|c|c|c|c|c|c|c|c|c|c|c|c|c|c|c|}
\hline Source of variation & df & $\begin{array}{l}\text { Days to } \\
50 \% \\
\text { flowering }\end{array}$ & $\begin{array}{l}\text { Plant } \\
\text { height } \\
\text { (cm) }\end{array}$ & $\begin{array}{l}\text { Effective } \\
\text { tillers }\end{array}$ & $\begin{array}{l}\text { Panicle } \\
\text { length( } \\
\text { cm) }\end{array}$ & $\begin{array}{l}\text { Flag leaf } \\
\text { length } \\
(\mathrm{cm})\end{array}$ & $\begin{array}{l}\text { Flag leaf } \\
\text { width } \\
(\mathrm{cm})\end{array}$ & $\begin{array}{l}\text { Panicle } \\
\text { weight (g) }\end{array}$ & $\begin{array}{l}\text { Panicle } \\
\text { density }\end{array}$ & $\begin{array}{l}\text { Filled } \\
\text { grains per } \\
\text { panicle }\end{array}$ & $\begin{array}{l}\text { Test } \\
\text { weight }(\mathrm{g})\end{array}$ & $\begin{array}{l}\text { Grain } \\
\text { yield } \times \\
\text { plant }(\mathrm{g})\end{array}$ & $\begin{array}{l}\text { Straw yield } \\
\times \text { plant }(\mathrm{g})\end{array}$ & HI \\
\hline Replication & 1 & 1.68 & 6.54 & 0.125 & 0.306 & 1.003 & 0.13 & 0.69 & 0.016 & 4.0134 & 0.073 & 18.0 & 0.18 & 0.00005 \\
\hline Genotypes & 35 & $83.3 * *$ & $215.3 * *$ & $14.66^{* *}$ & $11.72 * *$ & $30.48 * *$ & $0.12 * *$ & $2.99 * *$ & $9.32 * *$ & $6423.99 * *$ & $22.72 * *$ & $119.81 * *$ & $162.93 * *$ & $0.016 * *$ \\
\hline GCA & 7 & $75.5^{* * *}$ & $96.4 * *$ & $4.38 * *$ & $6.90 * *$ & $22.4 * *$ & $0.07 * *$ & $1.32 * *$ & $4.83 * *$ & $2988.90 * *$ & $13.63 * *$ & $56.08 * *$ & $35.98 * *$ & $0.0002 * *$ \\
\hline SCA & 28 & $33.2 * *$ & $110.4 * *$ & $8.067 * *$ & $5.59 * *$ & $13.4 * *$ & $0.06 * *$ & $1.54 * *$ & $4.62 * *$ & $3267.77 * *$ & $10.79 * *$ & $60.86 * *$ & $92.38 * *$ & $0.009 * *$ \\
\hline Parents & 7 & $68.2 * *$ & $349.4 * *$ & $9.96 * *$ & $18.2 * *$ & $38.99 * *$ & $0.11 * *$ & $2.64 * *$ & $14.22 * *$ & $8730.96 * *$ & $29.33 * *$ & $108.30 * *$ & $6.11 * *$ & $0.013 * *$ \\
\hline Crosses & 27 & $83.4 * *$ & $156.1 * *$ & $16.27 * *$ & $9.38 * *$ & $27.83^{* *}$ & $0.12 * *$ & $3.17 * *$ & $8.16 * *$ & $6053.26 * *$ & $17.65 * *$ & $126.7 * *$ & $3.29 * *$ & $0.015^{* *}$ \\
\hline Parents vs Hybrids & 1 & $186.0 * *$ & $873.2 * *$ & 4.06 & $29.6 * *$ & $42.0 * *$ & 0.00 & $0.706^{* *}$ & $6.44 * *$ & 285.01 & $113.33 * *$ & 13.76 & $89.78 * *$ & $0.035 * *$ \\
\hline Error & 35 & 2.22 & 10.59 & 2.01 & 0.78 & 1.54 & 0.02 & 0.05 & 0.21 & 140.699 & 0.2900 & 4.26 & 0.036 & 0.00112 \\
\hline${ }^{2} \mathbf{G C A}$ & & 7.44 & 9.10 & 0.337 & 0.65 & 2.16 & 0.006 & 0.129 & 0.473 & 291.855 & 1.348 & 5.39 & 169.87 & 0.00022 \\
\hline${ }^{2} \mathrm{SCA}$ & & 32.11 & 105.2 & 7.06 & 5.21 & 12.7 & 0.046 & 1.517 & 4.51 & 3197.42 & 10.64 & 58.73 & 158.06 & 0.00863 \\
\hline $\begin{array}{l}{ }^{2} \mathbf{G C A} / \\
{ }^{2} \mathrm{SCA}\end{array}$ & & 0.231 & 0.09 & 0.047 & 0.125 & 0.17 & 0.131 & 0.09 & 0.1048 & 0.091 & 0.126 & 0.091 & 246.03 & 0.025 \\
\hline
\end{tabular}

Table.1 Contd....

\begin{tabular}{|c|c|c|c|c|c|c|c|c|c|c|c|c|c|}
\hline Sourceof variation & df & $\begin{array}{l}\text { Hulling } \\
\text { percent }\end{array}$ & $\begin{array}{l}\text { Milling } \\
\text { percent }\end{array}$ & $\begin{array}{l}\text { Head rice } \\
\text { recovery }\end{array}$ & $\begin{array}{l}\text { Kernel } \\
\text { length } \\
(\mathrm{mm})\end{array}$ & $\begin{array}{l}\text { Kernel } \\
\text { width } \\
\text { (mm) }\end{array}$ & L×B ratio & $\begin{array}{l}\text { Kernel } \\
\text { length after } \\
\text { cooking }\end{array}$ & $\begin{array}{l}\text { Kernel } \\
\text { width } \\
\text { after } \\
\text { cooking }\end{array}$ & $\begin{array}{l}\text { Kernel } \\
\text { elongation } \\
\text { ratio }\end{array}$ & $\begin{array}{l}\text { Volume } \\
\text { expansion } \\
\text { ratio }\end{array}$ & $\begin{array}{l}\text { Water } \\
\text { uptake }\end{array}$ & $\begin{array}{l}\text { Alkali } \\
\text { spreading } \\
\text { value }\end{array}$ \\
\hline Replication & 1 & 0.029 & 7.157 & 1.272 & 0.003 & 0.009 & 0.014 & 0.110 & 0.011 & 0.003 & 0.005 & $4851.125^{* *}$ & 0.376 \\
\hline Genotype & 35 & $15.410 * *$ & $89.768 * *$ & $185.564 * *$ & $0.318 * *$ & $0.026 * *$ & $0.146 * *$ & $0.513 * *$ & $0.076 * *$ & $0.009 * *$ & $0.191 * *$ & $4177.068 * *$ & $3.386 * *$ \\
\hline GCA & 7 & $9.337 * *$ & $63.054 * *$ & $116.330 * *$ & $0.140 * *$ & $0.012 * *$ & $0.092 * *$ & $0.237 * *$ & $0.051 * *$ & 0.003 & $0.074 *$ & $2668.791 * *$ & $1.901 * *$ \\
\hline SCA & 28 & $7.297 * *$ & $40.341 * *$ & $86.895 * *$ & $0.164 * *$ & $0.013 * *$ & $0.068 * *$ & $0.261 * *$ & $0.035^{* *}$ & $0.005 * *$ & $0.101 * *$ & $1943.470 * *$ & $1.641 * *$ \\
\hline Parents & 7 & $24.882 * *$ & $197.037 * *$ & $273.590 * *$ & $0.036 * *$ & $0.313 * *$ & $0.712 * *$ & $0.662 * *$ & 0.027 & 0.009 & 0.088 & $5919.348 * *$ & $3.345^{* *}$ \\
\hline Hybrids & 27 & $12.195^{* *}$ & $56.935 * *$ & $167.692 * *$ & $0.025 * *$ & $0.104 * *$ & $0.224 * *$ & $0.491 * *$ & $0.090 * *$ & $0.010 * *$ & $0.218 * *$ & $3662.572 * *$ & $3.497 * *$ \\
\hline Parents vs Hybrids & 1 & $35.912 * *$ & $225.373 * *$ & $51.920 * *$ & 0.000 & $0.123 * *$ & 0.111 & 0.070 & 0.034 & 0.000 & 0.192 & $2872.509 * *$ & $0.660 *$ \\
\hline Error & 35 & 2.239 & 2.845 & 5.140 & 0.006 & 0.011 & 0.029 & 0.076 & 0.018 & 0.004 & 0.057 & 186.954 & 0.152 \\
\hline${ }^{2}$ GCA & & 0.822 & 6.163 & 11.376 & 0.0125 & 0.00 & 0.0086 & 0.0199 & 0.0042 & 0.000006 & 0.0045 & 257.5 & 0.182 \\
\hline${ }^{2} \mathrm{SCA}$ & & 6.177 & 38.92 & 84.32 & 0.149 & 0.0104 & 0.0629 & 0.223 & 0.0259 & 0.00307 & 0.0723 & 1849.9 & 1.565 \\
\hline $\begin{array}{ll}\sigma & { }^{2} \mathbf{G C A} / \\
\sigma & { }^{2} \mathbf{S C A}\end{array}$ & & 0.133 & 0.158 & 0.135 & 0.084 & 0.0862 & 0.1369 & 0.089 & 0.163 & 0.0204 & 0.0627 & 0.139 & 0.117 \\
\hline
\end{tabular}

$*$,** Significant at $5 \%$ and $1 \%$ level, respectively. 
Table.2 General combining ability of the parents for yield and quality traits

\begin{tabular}{|c|c|c|c|c|c|c|c|c|c|c|c|c|c|}
\hline Parents & $\begin{array}{l}\text { Days to } 50 \% \\
\text { flowering }\end{array}$ & $\begin{array}{l}\text { Plant } \\
\text { height } \\
\text { (cm) }\end{array}$ & $\begin{array}{l}\text { Effectiv } \\
\text { e tillers }\end{array}$ & $\begin{array}{l}\text { Panicle } \\
\text { length } \\
(\mathrm{cm})\end{array}$ & $\begin{array}{l}\text { Flag } \\
\text { leaf } \\
\text { length } \\
(\mathrm{cm})\end{array}$ & $\begin{array}{l}\text { Flag } \\
\text { leaf } \\
\text { width } \\
(\mathrm{cm})\end{array}$ & $\begin{array}{l}\text { Panicle } \\
\text { weight(g) }\end{array}$ & $\begin{array}{l}\text { Panicle } \\
\text { density }\end{array}$ & $\begin{array}{l}\text { Filled grains } \\
\text { per panicle }\end{array}$ & $\begin{array}{l}\text { Test } \\
\text { weight }(g)\end{array}$ & $\begin{array}{l}\text { Grain } \\
\text { yieldxplant } \\
\text { (g) }\end{array}$ & $\begin{array}{l}\text { Straw } \\
\text { yieldxplant } \\
\text { (g) }\end{array}$ & HI \\
\hline Kavya & $-1.438 * *$ & 0.149 & -0.188 & -0.114 & $-0.539 *$ & $\begin{array}{l}0.119 * \\
*\end{array}$ & $0.175^{* *}$ & $1.276^{* *}$ & $27.213 * *$ & $1.779 * *$ & $-2.38 * *$ & $-1.05^{*}$ & $-0.017^{*}$ \\
\hline BPT 5204 & $-2.688 * *$ & $-3.551 * *$ & 0.513 & $-0.814 * *$ & $-1.124 * *$ & $-0.066^{*}$ & 0.050 & $0.295 * *$ & 1.163 & 0.099 & $-1.025 *$ & $-3.4 * *$ & $-0.023^{* *}$ \\
\hline $\begin{array}{l}\text { Gontrabidha } \\
\text { n }\end{array}$ & $-2.088 * *$ & $1.384^{*}$ & -0.338 & $0.621 * *$ & 0.221 & 0.019 & $0.159 * *$ & 0.149 & 4.113 & $0.306^{*}$ & $3.12 * *$ & -0.63 & -0.006 \\
\hline R1556-2577 & $-3.088 * *$ & 1.129 & $-0.788 *$ & $1.071 * *$ & $0.861 * *$ & 0.044 & $0.376 * *$ & $0.359 * *$ & 1.813 & $1.411 * *$ & $1.82 * *$ & -0.53 & -0.004 \\
\hline MTU 1075 & $2.663 * *$ & $4.179 * *$ & $-0.938 * *$ & $0.656 * *$ & $1.856 * *$ & 0.039 & $0.234 * *$ & $0.536 * *$ & $17.113^{* *}$ & $0.606 * *$ & $2.055 * *$ & 0.275 & -0.003 \\
\hline WGL 915 & $1.313 * *$ & $-3.606^{* *}$ & $0.713^{*}$ & $-1.194 * *$ & $-1.189 * *$ & -0.036 & $0.398 * *$ & 0.054 & $-12.988 * *$ & $0.884 * *$ & $-2.76 * *$ & $1.350 *$ & $0.021 * *$ \\
\hline NLR 40024 & $1.217 * *$ & $3.409 * *$ & 0.263 & $0.496 *$ & $2.016^{* *}$ & 0.039 & $0.191 * *$ & $0.649 * *$ & $-11.138 * *$ & $1.451 * *$ & $2.61 *$ & $2.995 * *$ & $0.022 * *$ \\
\hline HKR08-62 & $4.713 * *$ & $-3.091 * *$ & $0.763 *$ & $-0.724 * *$ & $-2.104 * *$ & $\begin{array}{l}- \\
0.156^{*} \\
*\end{array}$ & $0.689 * *$ & $0.899 * *$ & $-27.288^{* *}$ & $1.014 * *$ & $-0.92 *$ & 0.99 & 0.010 \\
\hline
\end{tabular}

$*$, ** Significant at 5\% and $1 \%$ level, respectively.

Table.2 Contd.....

\begin{tabular}{|c|c|c|c|c|c|c|c|c|c|c|c|c|}
\hline Parents & $\begin{array}{l}\text { Hulling } \\
\text { percent }\end{array}$ & $\begin{array}{l}\text { Milling } \\
\text { percent }\end{array}$ & $\begin{array}{l}\text { Head rice } \\
\text { recovery } \\
(\%)\end{array}$ & $\begin{array}{l}\text { Kernel } \\
\text { length } \\
(\mathrm{mm})\end{array}$ & $\begin{array}{l}\text { Kernel } \\
\text { width } \\
\text { (mm) }\end{array}$ & $\begin{array}{l}\text { length } \times \text { bre } \\
\text { adth ratio }\end{array}$ & $\begin{array}{l}\text { Kernel } \\
\text { length } \\
\text { after } \\
\text { cooking }\end{array}$ & $\begin{array}{l}\text { Kernel } \\
\text { width after } \\
\text { cooking }\end{array}$ & $\begin{array}{l}\text { Elongation } \\
\text { ratio }\end{array}$ & $\begin{array}{l}\text { Volume } \\
\text { expansion } \\
\text { ratio }\end{array}$ & $\begin{array}{l}\text { Water } \\
\text { uptake }\end{array}$ & $\begin{array}{l}\text { Alkali } \\
\text { spreading } \\
\text { value }\end{array}$ \\
\hline Kavya & $1.193 * *$ & $2.625 * *$ & $2.099 * *$ & -0.071 & $-0.034 *$ & $0.053^{*}$ & $-0.183^{* *}$ & $-0.065^{*}$ & -0.022 & -0.053 & $-29.238 * *$ & $-0.683 * *$ \\
\hline BPT 5204 & -0.603 & $-1.207 * *$ & $-2.389 * *$ & $-0.189 * *$ & -0.005 & $-0.072 * *$ & $-0.133 *$ & -0.035 & 0.016 & -0.063 & 4.413 & 0.008 \\
\hline Gontrabidhan & -0.194 & 0.031 & $-4.253 * *$ & 0.004 & $0.033 *$ & $-0.082 * *$ & 0.052 & $0.093 * *$ & 0.012 & -0.083 & $16.413 * *$ & $0.498 * *$ \\
\hline R1556-2577 & $0.987 * *$ & $-0.895^{*}$ & $-2.626 * *$ & $0.139 * *$ & -0.015 & $0.093 * *$ & $0.172 * *$ & -0.023 & 0.002 & -0.053 & $7.863 * *$ & 0.098 \\
\hline MTU 1075 & $-1.892 * *$ & $-5.001 * *$ & $-3.037 * *$ & $0.093 *$ & $-0.047 * *$ & $0.162 * *$ & -0.031 & $-0060 *$ & -0.023 & 0.098 & $-20.288 * *$ & $-0.443 * *$ \\
\hline WGL 915 & 0.381 & $2.822 * *$ & $2.923 * *$ & $-0.089 *$ & 0.027 & $-0.112 * *$ & $-0.142 *$ & $0.080 * *$ & -0.010 & 0.023 & 0.413 & $0.383 * *$ \\
\hline NLR 40024 & -0.006 & $1.401 * *$ & $4.649 * *$ & $-0.144 * *$ & $0.053 * *$ & 0.002 & $0.248 * *$ & $0.080 * *$ & 0.018 & $0.158 * *$ & $14.813 * *$ & $0.428 * *$ \\
\hline HKR08-62 & 0.134 & 0.223 & $2.637 * *$ & -0.029 & -0.011 & -0.042 & 0.016 & $-0.070^{*}$ & 0.007 & -0.028 & 5.613 & $-0.288 * *$ \\
\hline
\end{tabular}


Int.J.Curr.Microbiol.App.Sci (2018) 7(1): 2834-2843

Table.3 Specific combining ability effects of hybrids for yield, yield components and quality traits in rice

\begin{tabular}{|c|c|c|c|c|c|c|c|c|c|c|c|c|c|}
\hline Hybrid & $\begin{array}{l}\text { Days to 50\% } \\
\text { flowering }\end{array}$ & $\begin{array}{l}\text { Plant } \\
\text { height }(\mathrm{cm})\end{array}$ & $\begin{array}{l}\text { Bfíective } \\
\text { tillers }\end{array}$ & $\begin{array}{l}\text { Panicle } \\
\text { length(cm) }\end{array}$ & $\begin{array}{l}\text { Flag leaf } \\
\text { length }(\mathrm{cm})\end{array}$ & $\begin{array}{l}\text { Flag leaf } \\
\text { width }(\mathrm{cm})\end{array}$ & $\begin{array}{l}\text { Panicle } \\
\text { weight(g) }\end{array}$ & $\begin{array}{l}\text { Panicle } \\
\text { density }\end{array}$ & $\begin{array}{l}\text { Filled grains } \\
\text { per panicle }\end{array}$ & $\begin{array}{l}\text { Test } \\
\text { weight(g) }\end{array}$ & $\begin{array}{l}\text { Grain } \\
\text { yield×plant(g) }\end{array}$ & $\begin{array}{l}\text { Straw } \\
\text { yield×plant(g) }\end{array}$ & HII \\
\hline Kavyax BPT5204 & $3.194 * *$ & 2.768 & 0.856 & 1.040 & 0.592 & 0.141 & $0.805^{* *}$ & $1.368^{* *}$ & $46.528^{* * *}$ & 0.168 & $7.551 * *$ & 2.183 & 0.013 \\
\hline Kavyax Gontrabidhan & $-5.406^{* *}$ & $-10.167^{* *}$ & -1.294 & $-1.595^{* *}$ & -0.353 & -0.094 & $-0.958 * *$ & $-1.987 * *$ & $-60.422^{* *}$ & $1.257^{* *}$ & $-10.444^{* *}$ & -2.287 & -0.019 \\
\hline KavyaxR1556-2577 & $-2.406^{*}$ & -0.512 & -0.344 & -0.445 & -1.143 & -0.019 & -0.236 & $-0.177 * *$ & -4.122 & -0.548 & -1.644 & $-5.587^{* * *}$ & -0.036 \\
\hline KavyaxMTU1075 & -0.556 & 2.338 & $-2.194 *$ & 1.120 & 1.662 & 0.086 & $1.117^{* * *}$ & -0.372 & $-21.422^{* *}$ & $1.707^{* * *}$ & $9.231^{* *}$ & $4.708 * *$ & -0.017 \\
\hline KavyaxWGL915 & $-8.306^{* *}$ & $-7.677^{* * *}$ & -1.844 & -0.830 & -1.543 & -0.089 & $-0.482^{* *}$ & -0.032 & $-15.822^{*}$ & $-1.403 * *$ & 1.936 & $-7.767 * *$ & -0.006 \\
\hline KavyaxNLR40024 & $2.294 *$ & -0.792 & -0.894 & 1.080 & 0.252 & 0.136 & -0.181 & -0.437 & 2.828 & $1.562^{* * *}$ & 0.686 & -2.812 & $-0.082^{* *}$ \\
\hline KavyaxHKR08-62 & 1.294 & 1.308 & $4.606^{* *}$ & 0.350 & $3.022^{* *}$ & 0.031 & $1.104 * *$ & $2.963^{* *}$ & $77.478^{* *}$ & -0.523 & $14.096 * *$ & 3.093 & 0.035 \\
\hline BPT5204×Gontrabidhan & -1.156 & $-9.367^{* * *}$ & 0.006 & -0.645 & -0.118 & -0.059 & 0.002 & $2.943 * *$ & $57.625 * *$ & $-4.573^{* *}$ & $3.011^{*}$ & 0.863 & -0.002 \\
\hline BPT5204× R1556-2577 & $8.344^{* *}$ & $16.488 * *$ & $-3.044^{* *}$ & $1.855^{* *}$ & $8.992^{* *}$ & $0.516^{* *}$ & $1.664^{* *}$ & $1.553^{* *}$ & $57.428^{* *}$ & 0.273 & $8.811^{* *}$ & $16.363 * *$ & $0.150^{* *}$ \\
\hline BPT5204×MTU1075 & $-7.806 * *$ & $-14.762 * *$ & -0.394 & $-3.180^{* * *}$ & $-6.353 * *$ & -0.029 & $-1.228 * *$ & -0.292 & $-36.372 * *$ & -0.623 & $-7.714 * *$ & $-7.342 * *$ & $-0.075^{* *}$ \\
\hline BPT5204× WGL915 & $1.944^{*}$ & $8.823 * *$ & $5.456^{* *}$ & $3.770^{* *}$ & 0.842 & $0.246^{* *}$ & $1.343^{* *}$ & $-1.302 * *$ & 1.228 & $3.467^{* *}$ & $5.591^{* *}$ & $5.283 * *$ & 0.035 \\
\hline BPT5204x NLR40024 & 1.044 & -0.692 & -1.594 & $-1.820^{* *}$ & $-3.563^{* *} *$ & $-0.429^{* * *}$ & $-0.921^{* *}$ & $-0.957^{* *}$ & $-38.122 * *$ & -0.268 & 2.241 & $-5.262 * *$ & $-0.056^{*}$ \\
\hline BPT5204× HKR08-62 & -1.456 & $9.808^{* *}$ & $-3.594^{* *}$ & 0.700 & 1.557 & -0.034 & $0.614^{* *}$ & $1.493^{* * *}$ & $40.528^{* * *}$ & $-1.003 * *$ & $-15.299^{* *}$ & $-8.357^{* * *}$ & $-0.093 * *$ \\
\hline Gontrabidhanx R1556-2577 & 1.244 & $-7.647^{* *}$ & -0.194 & $-3.480^{* *}$ & $-2.353 * *$ & $-.369^{* *}$ & $-1.499 * *$ & -0.152 & $-37.022 * *$ & $-3.383^{* *}$ & $-14.284^{* *}$ & $-11.407^{* *}$ & $-0.117 * *$ \\
\hline Gontrabidhanx MTU1075 & $5.094^{* * *}$ & $5.603^{*}$ & -0.544 & 0.435 & $-2.848^{* *}$ & -0.114 & 0.193 & -0.247 & 0.822 & $-0.728^{*}$ & $6.091^{* *}$ & 1.188 & 0.018 \\
\hline Gontrabichanx WGL915 & 1.344 & $-5.362^{*}$ & $-4.194^{* *}$ & $-1.215^{*}$ & 0.847 & $0.361^{\text {** }}$ & $-1.250^{* *}$ & $-1.857 * *$ & $-54.222 * *$ & $1.062^{* *}$ & $-5.004 * *$ & 23.613** & $0.228^{* * *}$ \\
\hline Gontrabidhanx NLR40024 & $-4.556^{* *}$ & -2.227 & 1.256 & $2.545^{* *}$ & -0.308 & 0.136 & $1.171^{* *}$ & $-1.012 * *$ & -10.072 & $5.277^{* *}$ & 1.146 & $-11.032^{* *}$ & $-0.103^{* *}$ \\
\hline Gontrabidhanx HKR08-62 & $-8.056^{* *}$ & $-10.927^{* *}$ & $4.256^{* * *}$ & $-1.185^{*}$ & -1.338 & 0.031 & $-1.074 * *$ & $-2.362 * *$ & $-67.422 * *$ & 0.442 & $10.156^{* *}$ & -3.727 & -0.035 \\
\hline R1556-2577× MTU1075 & $-3.406 * *$ & 2.958 & $-2.094^{*}$ & 0.985 & 0.112 & -0.039 & $2.116^{* *}$ & $2.113^{* *}$ & $69.478 * *$ & $2.467 * *$ & $-6.409^{* *}$ & $-4.812^{* *}$ & -0.040 \\
\hline R1556-2577x WGL915 & $-2.656^{* *}$ & $-7.057^{* * *}$ & -1.244 & -0.565 & $-2.843 * *$ & -0.064 & $-0.398 * *$ & $1.803^{* *}$ & $40.578^{* *}$ & $-4.643 * *$ & $-5.004 * *$ & $-9.987 * *$ & $-0.109^{* *}$ \\
\hline R1556-2577x NLR40024 & $-2.556^{*}$ & $5.928 * *$ & -1.294 & 1.145 & -0.048 & 0.061 & $1.348^{* * *}$ & $2.248^{* *}$ & $69.228^{* * *}$ & $-2.428 * *$ & $-4.554 * *$ & $-6.432^{* *}$ & $-0.060^{* *}$ \\
\hline R1556-2577 × HKR08-62 & $-5.506^{* *}$ & $6.028^{* *}$ & -0.294 & $1.265^{*}$ & 0.472 & $0.206^{*}$ & $0.638^{* *}$ & 0.202 & 2.878 & $1.437^{*}$ & $3.656^{* *}$ & $-2.927^{*}$ & -0.028 \\
\hline 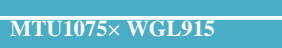 & $-8.306^{* *}$ & $-11.407^{* *}$ & $1.906^{*}$ & $-1.450^{* *}$ & $-7.238^{* *}$ & $-0.459 * *$ & $-1.170^{* * *}$ & $-2.842^{* *}$ & $-75.222 * *$ & -0.138 & 0.671 & $-11.642^{* *}$ & $-0.115^{* *}$ \\
\hline MTU1075x NLR40024 & 0.794 & 1.578 & $4.356^{* *}$ & $-2.440^{* *}$ & $5.757^{* *}$ & $0.216^{*}$ & -.254 & $4.653^{* *}$ & $89.928^{* * *}$ & $-7.673 * *$ & -2.179 & $8.263^{* *}$ & $0.099^{* *}$ \\
\hline MTU1075x HKR08-62 & $8.294^{* * *}$ & $-8.422^{* *}$ & 0.856 & $-2.670^{* *}$ & 0.677 & 0.011 & $-1.089 * *$ & 0.553 & 9.922 & $-5.358^{* *}$ & $-8.219^{* *}$ & $11.468^{* *}$ & $0.122 * *$ \\
\hline WGL915× NLR40024 & $-4.956^{* *}$ & $-9.837^{* * *}$ & $3.206^{* *}$ & $-1.890^{* *}$ & 0.398 & -0.109 & $-1.058 * *$ & $-2.157 * *$ & $-61.472 * *$ & $0.717 *$ & -2.474 & $-5.862^{* *}$ & $-0.060^{* *}$ \\
\hline WGL915x $\mathrm{HIKR} 018-62$ & $13.044^{* *}$ & $10.763^{* * *}$ & -0.794 & $2.880^{* *}$ & 1.322 & $0.186^{*}$ & -0.078 & -0.307 & $18.178^{*}$ & $-2.368^{* *}$ & $2.736^{*}$ & $11.293 * *$ & $0.107 * *$ \\
\hline NLR40024× HKR08-62 & $-5.356 * *$ & $-19.652^{* *}$ & $2.656^{* * *}$ & $-5.360^{* *}$ & $-7.083^{* *}$ & $-0.439 * *$ & $-1.722^{* * *}$ & -0.512 & $-51.672 * *$ & $-2.953^{* *}$ & -0.914 & $-8.752^{* *}$ & $-0.084 * *$ \\
\hline
\end{tabular}


Int.J.Curr.Microbiol.App.Sci (2018) 7(1): 2834-2843

Table.3 Contd......

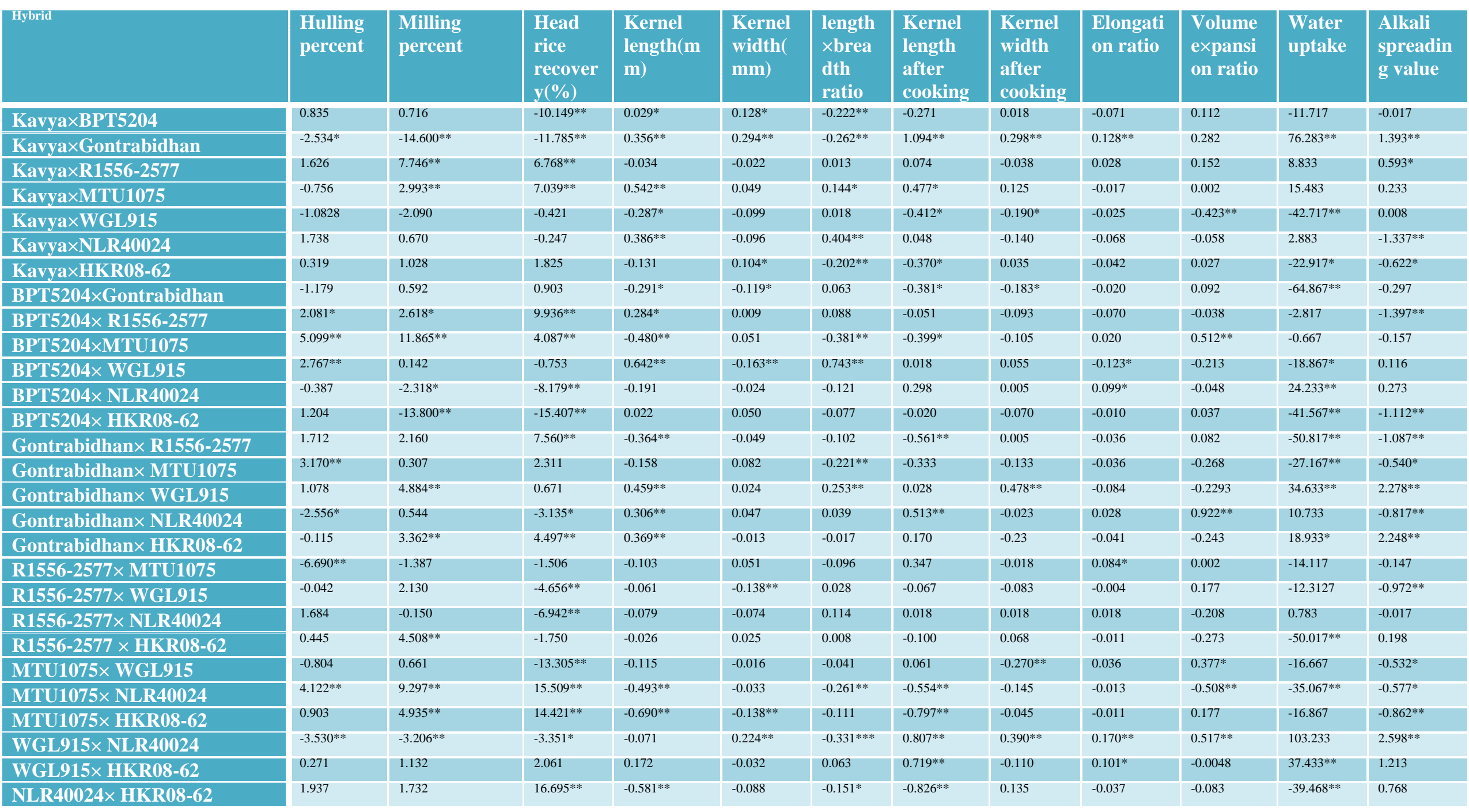

*, ** Significant at $5 \%$ and $1 \%$ level, respectively. 
Among the nine hybrids, five hybrids have at least one parent with significant positive GCA effect. The hybrid Kavya $\times$ HKR08-62 showed significant positive SCA effects for effective tillers per plant, panicle weight, panicle density and filled grains per panicle. The hybrid Gontrabidan $\times$ HKR08-62 for days to $50 \%$ flowering and plant height in desired direction, effective tillers per plant, milling percent, head rice recovery, kernel length, water uptake and alkali spreading value, while Kavya $\times$ MTU 1075 and BPT $5204 \times$ MTU 1075 and BPT $5204 \times$ WGL 915 recorded significant positive effects in desired direction for six characters each. BPT $5204 \times$ WGL 915 was registered high significant effects for effective tillers, panicle length, panicle weight, test weight, straw yield / plant, hulling percent, kernel length and kernel width in desired direction and length / breadth ratio. However, no cross combined all values in a desirable direction indicating the necessity of previous breeding value of the parents to combine desirable SCA effects in a single hybrid. The superiority of the cross may be due to complementary and duplicate type of gene interactions. Majority of the crosses with high SCA effects involved with high $\times$ low combinations indicating additive $x$ dominance, dominance $\times$ dominance type of gene interactions for expression of traits. Similar results were earlier reported by Rukmini Devi et al., (2017 b), Vanaja et al., (2003) and Gnanamalar and Vivekanandhan (2013). For days to $50 \%$ flowering negative amount of SCA are good and best crosses were Kavya × WGL 915, MTU 1075 × WGL 915 and Gontrabidhan $\times$ HKR08-62. Positive and significant estimates of SCA are desirable for head rice recovery and good specific combiners were NLR $40024 \times$ HKR08-62, MTU $1075 \times$ NLR 40024, MTU $1075 \times$ HKR08-62 and BPT $5204 \times$ R1556-2577. For plant height, semi dwarf is preferable to avoid lodging, hence negative estimates of SCA are desirable and good specific combiners were NLR $40024 \times$ HKR08-62, BPT $5204 \times$ MTU 1075, MTU $1075 \times$ WGL 915 and Gontrabidhan $\times$ HKR08-62.Good specific combiners for effective tillers per plant were BPT $5204 \times$ WGL 915, Kavya $\times$ HKR08-62, MTU $1075 \times$ WGL 915, Gontrabidhan $\times$ HKR08-62, MTU $1075 \times$ NLR 40024, WGL $915 \times$ NLR 40024 and NLR $40024 \times$ HKR0862 ; for panicle length best specific combiners were BPT $5204 \times$ WGL 915, Gontrabidhan $\times$ NLR 40024, BPT $5204 \times$ R1556-2577 and WGL $915 \times$ HKR08-62 ; for milling percent best specific combiners were BPT $5204 \times$ MTU 1075, MTU 1075×NLR 40024,Gontrabidhan $\times$ WGL915, Kavya $\times$ MTU 1075 and BPT $5204 \times$ R1556-2577; for kernel width and kernel width after cooking negative estimates of SCA are desirable. The good specific combiners for kernel width are BPT 5204 × WGL 915, R1556-2577 × WGL915 and BPT5204 $\times$ Gontrabidhan, while MTU1075 $\times$ WGL-915 and BPT $5204 \times$ Gontrabidhan for kernel width after cooking. For the character elongation ratio WGL $915 \times$ NLR 40024, Kavya $\times$ Gontrabidhan, WGL $915 \times$ HKR08-62, R1556-2577 × MTU 1075 recorded significant SCA estimates in desirable direction.

In these hybrids all kinds of parental combinations like high $\mathrm{x}$ high, high $\mathrm{x}$ low, medium $\mathrm{x}$ medium and medium $\times$ low were found. In contrast to the GCA effects, the SCA effects represents dominance and epistatic component of variation and that is not fixable in nature. This suggests that either additive $\times$ additive or additive $\times$ dominance genetic interaction were predominant. An interaction between positive and positive allele in crosses involving high $\mathrm{x}$ high combiners which can be fixed in subsequent generation if no repulsion phase linkages are involved was reported by Adilaxmi and Raghava Reddy (2011) and Shivani et al., (2009). If crosses showing high SCA effect 
involved either both or one good general combining parents and they can be successfully exploited for varietal improvement and expected to through stable performing transgressive segregants carrying fixable gene effects. In crosses with medium $x$ low GCA effects, the high positive SCA effect may be due to the dominant $\mathrm{x}$ recessive interaction expected to produce desirable segregants in subsequent generation (Lingham, 1961).

In many crosses however, the crosses of high $x$ high GCA lead to inferior hybrids for many studied traits i.e., Gontrabidhan $\times$ R1556-2577, Gontrabidhan $\times$ NLR 40024, MTU $1075 \times$ NLR 40024 , for grain yield / plant and also many studied traits indicating epistatic gene action controlling for traits. Similar results were earlier reported by Rukmini Devi et al., (2014 a) Gnanamalar and Vivekanandhan (2013) and Rashmi et al., (2010). Verma and Srivastava (2004) and Vanaja et al., (2003), also reported that several traits in rice were controlled by epistatic gene effect. Therefore these crosses are expected to produce desirable segregants and could be exploited successfully in varietal improvement programme.

Interestingly the hybrids between slender and bold grain parents possess desirable significant SCA effects for quality traits besides grain yield. BPT $5204 \times$ R1556-2577 for hulling percent milling percent, head rice recovery and kernel length and kernel width in desired direction and length/breadth ratio respectively. In few crosses i.e. Kavya $\times$ BPT 5204, BPT 5204×WGL 915, WGL $915 \times$ HKR08-62 showing low $\times$ low general combiners showed high SCA suggesting the epistatic gene action may be due to genetic diversity in the form of heterozygous loci. It is also revealed that the poor $\mathrm{x}$ good general combiners exhibit high SCA have to be improved through population improvement, but in crosses having high SCA due to poor $\mathrm{x}$ poor general combiners may be exploited for heterosis breeding.
In conclusion present study revealed the importance of both additive and non-additive gene effects in governing yield, its related components and quality traits with predominance of non-additive gene action for most of the yield and quality traits. In this situation where both non additive and additive components were important for the expression of characters, especially where former component is predominant single pedigree method of selection would be effective for its improvement. To explore both additive and non-additive type of gene action for grain yield and grain quality traits, it was suggested to post pone the selection to later generations in pedigree breeding programme. At the same time population improvement programmes like reciprocal recurrent selection which may allow to accumulate the fixable gene effects as well as to maintain considerable variability and heterozygosity for exploiting non fixable gene effects will prove to be the most effective method (Joshi, 1979). However rice is a highly self-pollinated crop forming a single seed per pollination, this selection procedure is not practicable. So the possible choice is the use of biparental mating among selected crosses or use of selection procedure such as diallel selective mating (Jensen,1970) to be adopted in selecting crosses or characters in varietal improvement which will exploit both the additive and nonadditive gene components. Among the parents NLR 40024 was adjudged as the best combiner followed by HKR08-62 could be utilized in hybridization programmes because of their significant general combining ability for yield, number of yield components and quality traits. Though different parents had been found to be good general combiners for different characters, the results indicated that there was close relationship between mean performance of the parents and their GCA effects in most of the cases studied. The Hybrids namely Kavya $x$ HKR08-62, Gontrabidhan $\times$ HKR08-62, R1556-2577 × HKR 08-62 and BPT $5204 \times$ R1556-2577 could be utilized for hybrid rice programmes for selecting favorable segregants from segregating populations 


\section{References}

Adilakshmi, D., Raghava Reddy, P., 2011. Genetic analysis for grain quality traits in rice Oryza sativa L. Andhra Agriculture Journal 58(2), 147-150.

Fischer, S., Mohring, J., Schon, C.C., Piepho, H.P., Klein, D., Schipprack, W., Utz, H.F., Melchinger, A.E., Reif, J.C., 2008. Trends in genetic variation components during 30 years of hybrid maize breeding at the University of Hohenheim. Plant Breeding, 127, 446-451.

Gnanamalar, R.P., Vivekanandan, 2013. Combining ability analysis of grain quality traits in rice oryza sativa. Asian Journal of Plant Science and Research 3(2), 145-149.

Griffing, B., 1956. Concept of general and specific combining ability in relation to diallel crossing systems Australian Journal of Biological Science. 9, 463-493.

Jensen, N. F., 1970. A diallel selective mating system for cereal breeding. Crop Science, 10, 629-635.

Joshi, A. B., 1979. Breeding methodology for autogamous crops. Indian Journal of Genetics and plant breeding. 39, 567-578.

Lingham, D. C., 1961. The high low method of improvement. Crop Science, 1, 376-78.

Murthy, P.S.N., Govindaswamy, S., 1967. Inheritance of grain size and its correlation with the hulling and cooking qualities. Oryza, 4(1), 12-21.

Rashmi, M., Rabiei, B., Samizadeh-Lahiji and Kafi Ghasemi, A., 2010. Evaluation of combining ability in ice cultivars based on second and fourth Griffing methods Journal of Science and Technology of
Agriculture and Natural Resources. 12(43), 129-141

Ravi Kishore, Archana Devi, Preeti kumara, Sakat Dwivedi Ranjan Dwivedi., Giri, S.P., Dwivedi, D.K., Uppandey., 2017. Gene action and combining ability in rice Oryza sativa $\mathrm{L}$ involving indica and tropical japonica genotypes. International Journal of Current Microbiology and Applied Sciences, 6(7), 8-16

Rukmini Devi, K., Cheralu, C., Venkanna, V., Srinivas, G., 2014 a. Combining ability studies for quality and yield traits in some restorer lines of rice (Oryza sativa L) The Andhra Agricultural Journal, 61(2), 304308.

Rukmini Devi, K., Lingaiah, N., Parimala, K., Venkanna, V., Hari, Y., Satish Chandra, B., 2017 b. Magnitude of heterosis and gene effects for yield and quality traits in rice (Oryza sativa L.) Electronic Journal of Plant Breeding, 8(3): 799-810

Shivani, D., Viraktamath, B.C., Shobha Rani, N., 2009. Combining ability for grain quality characters in indica/indica hybrids of rice. Oryza, 46; 152-155

Vanaja, T., Lukins, C. Babu., Ramakrishnan, V.V., Pushkaran, K., 2003. Combining ability analysis for yield and yield components in rice varieties of diverse origin. Journal of tropical Agriculture 41, 7-15

Verma, O. P., Srivastava, H.K., 2004. Genetic component and combining ability analysis in relation to heterosis for yield and associated traits using three diverse rice growing ecosystems, Field Crop Research, 88, 91-102

\section{How to cite this article:}

Rukmini Devi, K., V. Venkanna, B. Satish Chandra and Hari, Y. 2018. Gene Action and Combining Ability for Yield and Quality Traits in Rice (Oryza sativa L.) using Diallel Analysis. Int.J.Curr.Microbiol.App.Sci. 7(01): 2834-2843. doi: https://doi.org/10.20546/ijcmas.2018.701.338 